\title{
Reoccurring bovine anthrax in Germany on the same pasture after 12 years
}

Peter Braun ${ }^{1}$, Wolfgang Beyer², Matthias Hanczaruk ${ }^{3}$, Julia M. Riehm ${ }^{3}$, Markus Antwerpen ${ }^{1}$, Christian Otterbein ${ }^{4}$, Jacqueline Oesterheld ${ }^{1}$ and Gregor Grass ${ }^{1 *}$

${ }^{1}$ Bundeswehr Institute of Microbiology (IMB), Munich, Germany

${ }^{2}$ Department of Livestock Infectiology and Environmental Hygiene, Institute of Animal Science, University of Hohenheim, Stuttgart, Germany

${ }^{3}$ Bavarian Health and Food Safety Authority, Oberschleißheim, Germany

${ }^{4}$ Local Veterinarian Unit, District Rosenheim, Germany

*Correspondence: gregorgrass@bundeswehr.org; Tel.: +49-992692-3981

Keywords: Bacillus anthracis, anthrax, outbreak, phylogenetics, detection assay

Running title: Recurring bovine anthrax after 12 years

\begin{abstract}
The zoonotic disease anthrax caused by the endospore-forming bacterium Bacillus anthracis is very rare in Germany. In the state of Bavaria, the last case occurred in July of 2009 resulting in four dead cows. In August of 2021, the disease reemerged after heavy rains, killing one gestating cow. Notably, both outbreaks affected the same pasture, suggesting a close epidemiological connection. $B$.

anthracis could be grown from blood culture and the presence of both virulence plasmids (pXO1 and pXO2) were confirmed by PCR. Also, recently developed diagnostic tools enabled rapid detection of B. anthracis cells and nucleic acids directly in clinical samples. The complete genome of the strain isolated from blood, designated BF-5, was DNA-sequenced and phylogenetically grouped within the B.Br.CNEVA clade that is typical for European $B$. anthracis strains. The genome was almost identical to $B F-1$, the isolate of 2009 , separated only by three single nucleotide polymorphisms on the chromosome, one on plasmid pXO2 and three indel-regions. Further, B. anthracis DNA was detected by PCR from soil-samples taken from spots, where the cow had fallen onto the pasture. New tools based on phage receptor binding proteins enabled the microscopic detection and isolation of $B$. anthracis directly from soil-samples. These environmental isolates were genotyped and found to be SNP-identical to BF-1. Therefore, it seems that the BF-5 genotype is currently the prevalent one at the affected premises. The contaminated area was subsequently disinfected with formaldehyde.
\end{abstract}

\section{Introduction}

Bacillus anthracis, the causative agent of anthrax, resides dormant in soils as endospores. These spores can resurface after heavy rains [1] or e.g., by disturbances of animal burial-sites [2]. Typically, susceptible grazing mammals become infected by ingesting spore-contaminated soil. The anthrax pathogen is notorious for unexpectedly re-emerging after years or decades of inactivity at previous outbreak -sites [1]. Such instances include outbreaks in Sweden [2], Siberia [3] or Italy [4,5]. In Germany, anthrax is very uncommon. The last human infections in 2012 were associated with illicit drug-consumption of heroin allegedly contaminated with $B$. anthracis spores [6-8]. Animal cases are equally rare with small-scale bovine outbreaks recorded in 2009 [9], 2012 [10] and 2014 [11]. While these animal cases involved B. anthracis genotypes common for Germany, the human cases raised concern as genotypes involved were distinct from any known German isolate but closely related to strains from the Near and Middle East [12]. Likely, spores of this genotype were introduced via drugtrafficking activities involving contaminated by-products en route $[6,12]$. Rapid identification and 
genotyping of new outbreak isolates is thus of importance to differentiate natural, reoccurring outbreaks of domestic strains from deliberate release or accidental contamination.

Therefore, occurrence of bovine anthrax in August of 2021 raised initial alarm. However, this outbreak has affected the same premises as in 2009. Back then, four heifers had succumbed to the disease and one euthanized [13]. Now, a gestating cow fell with strong suspicion of anthrax.

The genome (BF-1) of the 2009 anthrax-outbreak has been published [9]. This genome is closely related with other isolates of the B-branch phylogeny of $B$. anthracis (B.Br. CNEVA) [14]. The B.Br CNEVA genotype seems to be typical for mountainous areas in central Europe from France [14] to Slovakia [14] and from Sweden [2] to Switzerland [15]. Also, to this group belongs a historical genome reconstructed from a microscopy-slide prepared in Germany in 1878 featuring $B$. anthracisinfected dried-up cow-blood [14]. In this report, we described and investigated a rare reoccurring German anthrax-outbreak in southern Bavaria. Because of the very close spatial occurrence of the $2009 / 2021$ outbreaks, the question arose, whether these involved identical or different $B$. anthracis strains. We analyzed the genome-sequence of the 2021 outbreak isolate in phylogenetic relation to closely related strains and offered conclusions on the origin of this $B$. anthracis strain.

\section{Material and Methods}

\section{Bacterial culture}

Strain, B. anthracis Sterne (positive control) [16] and B. cereus ATCC10987 (negative control) were grown on Columbia blood agar (Becton Dickinson, Heidelberg, Germany) or trimethoprimsulfamethoxazole-polymyxin blood agar (TSPBA) [17]. B. anthracis was chemically inactivated with $4 \%(v / v)$ Terralin PAA (Schülke \& Mayr GmbH, Norderstedt, Germany), as in [18]. Blood-samples were inactivated within a class III biological safety cabinet at the Bundeswehr Institute of Microbiology BSL-3 facility by adding $50 \mathrm{ml} 4 \%(\mathrm{v} / \mathrm{v})$ Terralin PAA to $0.5 \mathrm{ml}$ blood. After incubation at room temperature for $30 \mathrm{~min}$, samples were washed twice with phosphate-buffered saline (PBS).

\section{Initial carcass samples, diagnostic polymerase chain reaction for B. anthracis and microscopy}

Blood-samples from the left nostril of the cow-carcass were taken and transferred to the federal state veterinary laboratory for further analysis. Sample-culture was conducted on Columbia blood agar, and grown overnight at $37^{\circ} \mathrm{C}$. A single colony with typical growth morphology was used for DNA preparation (Qiagen, Hilden, Germany) and polymerase chain reaction (PCR) was performed for chromosomal and both virulence plasmids markers ( $\mathrm{pXO1}$ and pXO2) as described in the manufacturer's instructions (RealStar ${ }^{\circledR}$ Anthrax PCR Kit 1.0; Altona, Hamburg, Germany).

For direct PCR-based detection of B. anthracis in blood-samples, $100 \mu$ inactivated blood-sample were incubated at $95^{\circ} \mathrm{C}$ for $10 \mathrm{~min}$ to lyse cells and centrifuged. Aliquots of $5 \mu \mathrm{l}$ of the supernatant were then used as templates for $16 \mathrm{~S}$ rRNA SNP-PCR or 16S rRNA SNP RT-PCR performed as described in [19]. Alternatively, total nucleic acid extractions of blood-samples were used as templates. MasterPure Complete DNA and RNA Purification Kit (Lucigen, Middleton, WI, USA) was used for extraction of DNA and RNA from blood-samples according to the manufacturer's instructions for whole-blood-samples.

For microscopic detection of $B$. anthracis from blood-samples, receptor binding protein (RBP) derivative $\mathrm{RBP}_{\lambda 03 \Delta 1-120}$ was used. A volume of $0.5 \mathrm{ml}$ blood was inactivated, repeatedly washed with 
PBS and mixed with $1 \mu \mathrm{g}$ mCherry-RBP ${ }_{\lambda 03 \Delta 1-120}$ protein. Fluorescence microscopy was conducted as described in [18].

\section{Collection of soil-samples}

On September 6, 2021, soil-samples were collected from several spots near where the diseased cow had fallen and subsequently exuded spore-contaminated blood onto the pasture. Because of heavy rains in the area in the meantime $\left(>50 \mathrm{l} / \mathrm{m}^{2}\right)$, samples were collected from approx. $10 \mathrm{~cm}$ below the surface.

\section{Soil-sample analysis by $P C R$ and culturing of $B$. anthracis}

Soil-samples for PCR analysis were processed as described in [20]. Briefly, three aliquots of soilsamples were resuspended in sterile water with glass-beads $(\varnothing 5 \mathrm{~mm})$ and mixed overnight at room temperature. Two of the aliquots were spiked beforehand with spores of strain $B$. anthracis Sterne 34F2 for quantification. The suspensions were filtered through sterile gauze to remove soil particles and other rough materials. After centrifugation at $4000 \times \mathrm{xg}$ for $15 \mathrm{~min}$, the pellet was washed three times in sterile water and finally re-suspended in $5 \mathrm{ml}$ aquadest. This suspension was heated to $65-$ $70^{\circ} \mathrm{C}$ for $30 \mathrm{~min}$ to inactivate vegetative cells. Volumes of $250 \mu \mathrm{l}$ each were plated onto four semiselective agar plates (TSPBA) [21]. Plates were incubated overnight at $37^{\circ} \mathrm{C}$. Then, the bacterial lawn

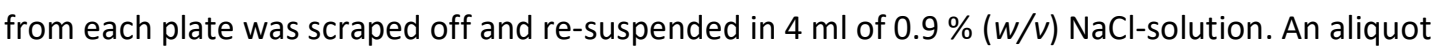
(ca. $1 \mathrm{ml}$ ) of this suspension was boiled for $20 \mathrm{~min}$ in a heating block to release DNA from cells, centrifuged at $12,000 \times \mathrm{xg}$ for $15 \mathrm{~min}$ and the supernatant filtered through a $0.45 \mu \mathrm{m}$ luer-lock filter. Aliquots of $5 \mu \mathrm{l}$ of the filtered supernatant were used for PCR analysis [20]. If PCR-positive, dilutions of the original suspension were plated and grown on TSPBA [17] for isolation and verification of suspicious $B$. anthracis colonies [20]. DNA from a picked colony was tested by PCR for anthrax markers as described in [1]. Additional enrichment of $B$. anthracis from soil-samples was achieved by culturing on semi-selective CEFOMA agar "Bacillus CEreus sensu lato group-specific antibiotics, FOsfomycin, Macrolides Agar" according to [22].

\section{Enrichment of B. anthracis from soil-samples by magnetic separation and culturing}

For enriching $B$. anthracis from possibly spore-contaminated soil-samples, a newly developed magnetic bead-assisted magnetic separation-method was applied. In this approach $\mathrm{RBP}_{\lambda 03 \Delta 1-120}$ [18] was re-purposed to capture B. anthracis from soil. In short, Strep-Tactin XT protein (IBA GmbH, Göttingen, Germany) was coupled to magnetic beads (Dynabeads ${ }^{T M} M-280$ Tosylactivated, ThermoFisher, Dreieich, Germany). Then $\mathrm{RBP}_{\lambda 03 \Delta 1-120}$ protein was attached to this Strep-Tactin XT via the Twin Strep-tag epitope. Soil was processed the same as described in [17], i.e., a soil-sample was shaken in PBS buffer with $0.5 \%(v / v)$ Tween 20 to solubilize spores. The sample was mildly centrifuged to remove solid material and the crudely cleared supernatant incubated at $62^{\circ} \mathrm{C}$ for 20 min to inactivate vegetative cells. The supernatant was mixed with Brain Heart Infusion broth (Merck, Darmstadt, Germany) with fetal calf serum (Merck) and incubated to allow spores to germinate and develop into vegetative cells. This germination culture was mixed and incubated with the RBP-loaded magnetic beads to separate B. anthracis spores from the liquid. Separation was accomplished using a magnetic stand (ThermoFisher). Beads were washed and finally plated onto 
TSPBA agar or Columbia blood agar plates (Becton Dickinson). Colonies were evaluated after incubating over night at $37^{\circ} \mathrm{C}$. Full details on the method will be published elsewhere.

\section{Rapid prescreening of candidate B. anthracis colonies}

Blood-samples from the carcass or colonies suspicious for B. anthracis obtained after enrichment from soil-samples, were subjected to colorimetric Enzyme-Linked Phage Receptor Binding Protein Assay (ELPRA) as described in [23]. In short, the one-step assay version was applied that utilizes recombinant HRP-coupled $\mathrm{RBP}_{\lambda 03 \Delta 1-120}$. Candidate colony material or blood was inactivated, washed twice with PBS and incubated with $0.1 \mu \mathrm{g}$ of HRP-RBP ${ }_{\lambda 03 \Delta 1-120}$ protein. Samples were repeatedly washed with PBS and the pellet resuspended in $50 \mu \mathrm{L}$ SeramunBlau ${ }^{\circledR}$ slow (containing 3,3',5,5'tetramethylbenzidin) peroxidase substrate (Seramun Diagnostica, Heidesee, Germany). Blue color development was monitored for several minutes and photo-documented.

\section{$D N A$ isolation from $B$. anthracis colony material and confirmative $P C R$}

Single bacterial colonies grown on semi-selective agar (TSPBA) were chemically inactivated with $4 \%$ Terralin PAA and DNA isolated using the MasterPure ${ }^{\mathrm{TM}}$ Gram Positive DNA Purification kit (Lucigen) with minor modifications as described in [24]. DNA concentrations were quantified using the Qubit dsDNA HS Assay Kit (Thermo Fisher Scientific), according to the supplier's protocol. For confirmation of B. anthracis DNA via PCR, the chromosomal marker dhp61 was used as described previously [25]. DNA preparations were stored at $-20^{\circ} \mathrm{C}$ until further use.

\section{Whole Genome Sequencing}

Nanopore sequencing was performed using SQK-LSK109 chemistry on a R10.3 SpotON Flow Cell on the GridION system (Oxford Nanopore Technologies, Oxford, UK) running system software MinKNOW 21.05.8. A total of 350,000 reads were generated using the implemented "super-accurate base calling" model. For increasing the assembly-efficacy the amount of reads were down-sampled to 104,110 reads (N50 of $10.01 \mathrm{~kb}$; mean raw quality score of Q13.5). After processing using Flye assembler V2.9 [26] three circularized high-quality replicons, corresponding to the chromosome (5,213,322 bp; coverage 174-fold) as well as both plasmids pXO1 (181,920 bp; coverage 614-fold) and pXO2 (94,735 bp; coverage 491-fold) were obtained. The scaffolds were manually checked for contaminant reads and annotated automatically by the NCBI Prokaryotic Genome Annotation Pipeline [27] after submission. All data generated or analyzed during this study are included in this published article, and its supplementary information files are publically available in the NCBI Sequence Read Archive (SRA) repository (Bioproject PRJNA309927). CanSNPer (v1.0.10) [28] was used to classify and subsequently assign the corresponding canSNP-group B.Br.CNEVA to this genome.

\section{Analysis of whole genome sequencing data and SNP-calling}

For rapid core chromosome multiple-alignment, the Parsnp tool from the Harvest Suite was used [29]. For this, a chromosome-dataset, representing genomes from public databases (Table S1) and the newly sequenced strains of $B$. anthracis, were aligned against the chromosome of $B$. anthracis 'Ames ancestor' (NC_007530) as a phylogenetic outgroup using Parsnp (parameters - $\mathrm{C}-\mathrm{e}-\mathrm{U}-\mathrm{C} 1000$ ). 
To export the identified SNP-positions, HarvestTools (version 1.0) from the same software suite was used to create a vcf-(Variant Calling File) listing all SNP-positions. In order to enhance data quality, chromosome regions with closely adjacent SNPs (<10 bp distance), and positions harboring undefined nucleotides ("N"), were removed. This curated vcf-file was used as input for HarvestTools to compile a multi-FASTA file out of the chromosome-dataset, comprising the concatenated SNPS as a multiple-sequence alignment. This concatenated sequence information was used to calculate a Maximum Likelihood tree in MEGA X [30,31]. A minimum spanning tree was computed in BioNumerics 6.6 (Applied Maths, Sint-Martens-Latem, Belgium) from the vcf SNP-file (in binary format) as input, and manually edited (using Powerpoint, Microsoft) for style.

Analysis of the distribution of SNPs specific for B. anthracis strain BF-5 in other isolates

DNA of several additional clones retrieved from soil sampling were subjected to SNP-analysis. For this, regions covering the SNP-regions identified by genome sequencing were PCR-amplified (Primers listed in Table S2) and Sanger DNA-sequenced (Eurofin Genomics, Ebersbach, Germany). DNAsequence analysis was conducted with Geneious Prime (Biomatters, USA).

\section{Results}

B. anthracis infection in a diseased cow was confirmed by initial in situ and PCR diagnostics

Veterinary examination of a diseased gestating cow on a pasture near Rosenheim (Bavaria, Germany) on August 24, 2021 raised suspicion of anthrax infection due to the disease-typical symptoms, i.e., sudden death and bloody discharge from all body orifices, including nostrils, eyes, vagina and anus (Figure $1 \mathrm{~A}, \mathrm{~B}$ ). PCR of DNA isolated from colonies with typical morphology grown after cultivating blood from the diseased animal gave positive results for diagnostic $B$. anthracis markers, the $d h p 61, p a g$, and cap genes, respectively (data not shown). Thus, anthrax disease was confirmed and an official diagnostic report released. 
A.

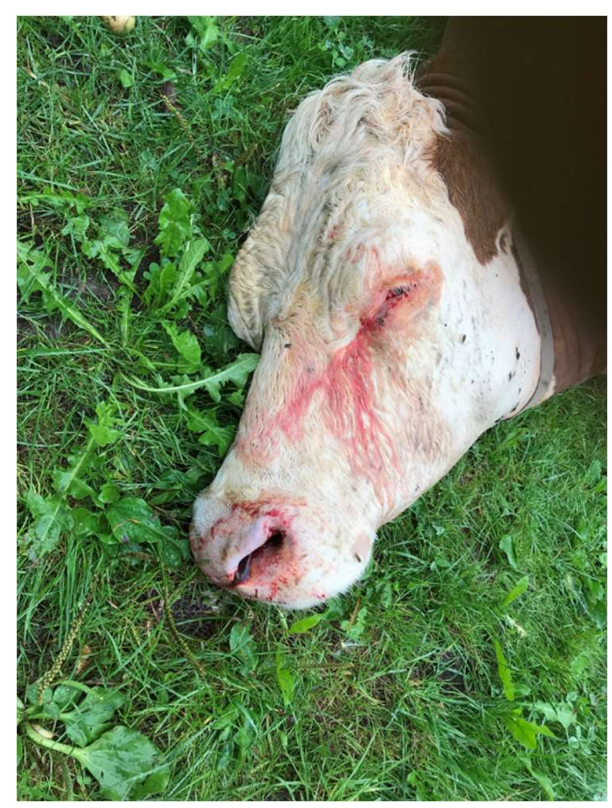

B.

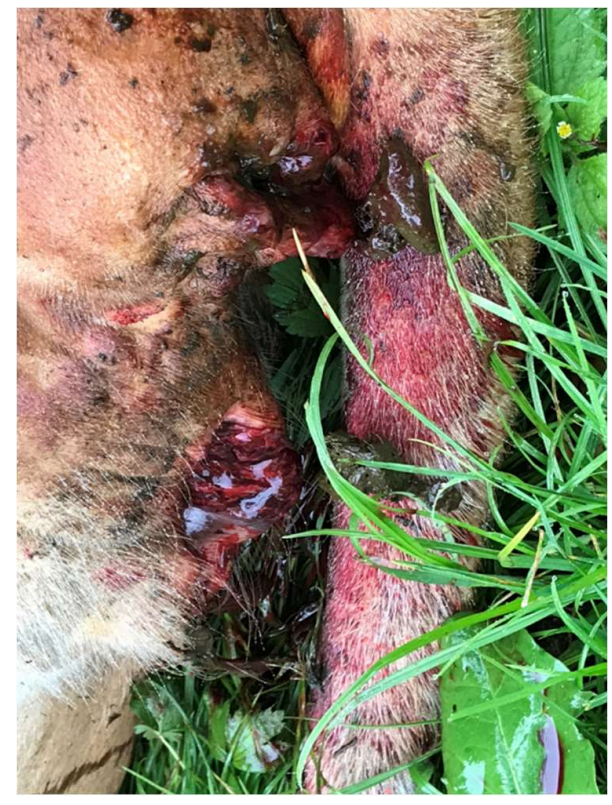

Figure 1: In situ and molecular PCR diagnostics of a cow diseased of anthrax. A two year old gestating cow fallen to anthrax on a pasture in southern Bavaria (Germany) in August of 2021 ( $A$ and $B)$. Close up of the head with bloody discharge out of eyes and left nostril (A) and rear view with bloody anus and vagina (B).

Detection of B. anthracis directly in blood-samples by phage RBP-based reporter and 16S rRNA SNP $(R T)-P C R$

Independent to initial diagnostic PCR analysis performed by state health authorities, blood taken from the left nostril of the carcass (Figure 1A) was subjected to recently developed ultrasensitive $16 \mathrm{~S}$ rRNA SNP (RT)-PCR [19] and phage RBP reporter-based rapid detection assays [18]. Results confirmed 
the previous PCR tests as phage $\mathrm{RBP}_{\lambda 03 \Delta 1-120}$ reporter based ELPRA gave positive results when inactivated blood-samples from the carcass were tested (Figure 2A). Using fluorescence microscopy, mCherry-RBP $\mathrm{X}_{\lambda 03 \Delta 1-120}$ reporter was found to specifically bind to bacterial chains in blood-sample as evidenced by red fluorescence (Figure $2 \mathrm{~B}$ ). This indicated that the detected cells were indeed very likely $B$. anthracis. Of note, these phage RBP-based tests can be performed in just a few minutes. Using 16S rRNA SNP-PCR, specific detection of $B$. anthracis nucleic acids directly in the blood-samples derived from the carcass as well as from nucleic acid extractions thereof, was also accomplished (Figure 2C). Dilutions (1:10 to 1:1000) of the inactivated blood-sample (without prior nucleic acid extraction) yielded $\mathrm{Ct}$ values from 24.9 to 31.7. Conversely, dilutions of total nucleic acid extracted from the same blood-sample yielded Ct values from 13.9 to 21.5 when testing for DNA only (Table S3). When these total nucleic acid preparations (containing DNA and RNA) were subjected to 16S rRNA SNP RT-PCR, the same samples (dilutions 1:10 to 1:1000) yielded even lower Ct values (9.7 to 17.8; Table S3). This is because the ultrasensitive RT version of the PCR not only detects 16S rRNA genes of $B$. anthracis but also their transcripts, which are more abundant in growing cells compared to their respective gene copies.

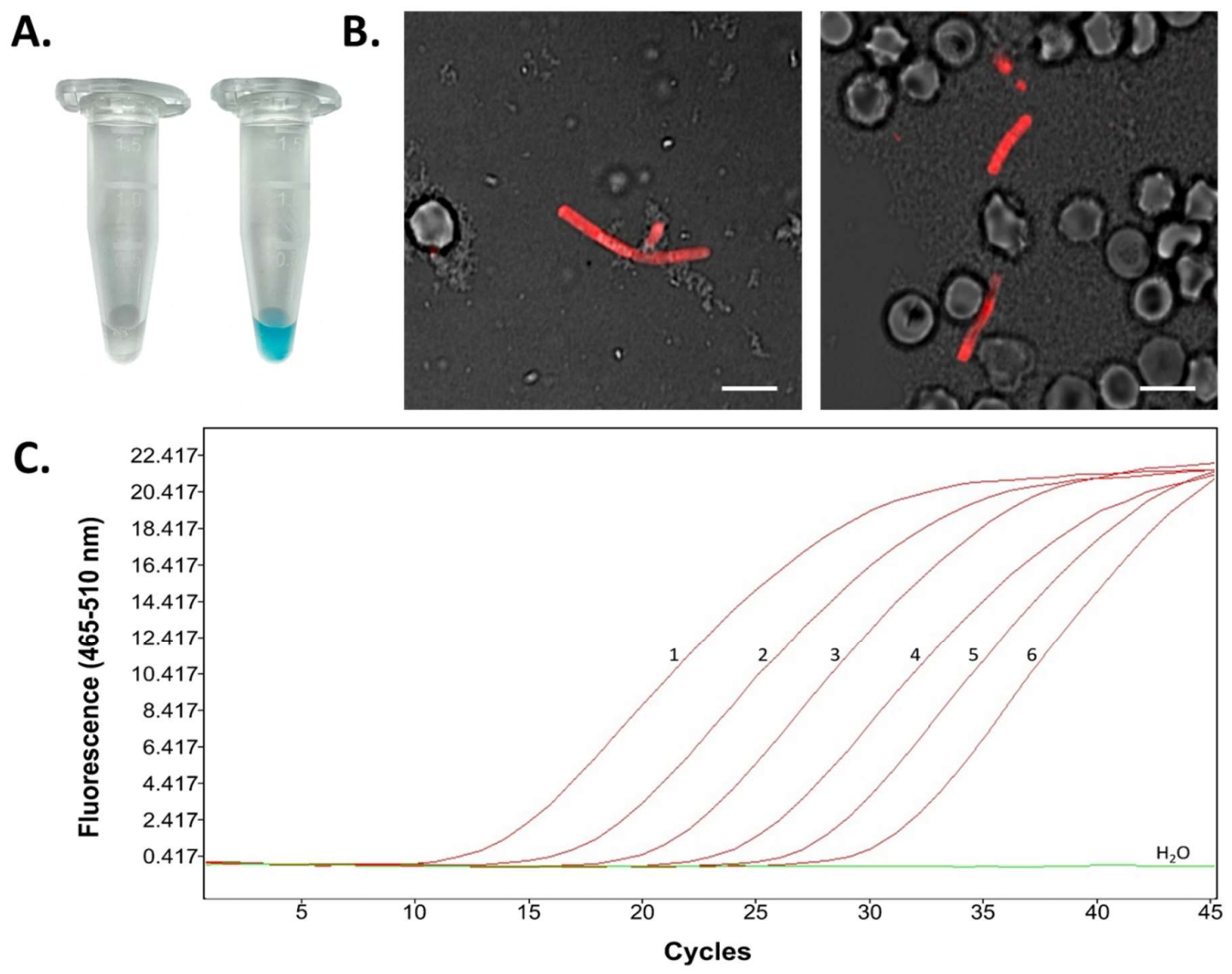

Figure 2: Direct detection of $\boldsymbol{B}$. anthracis cells in blood from a diseased cow. A: Horseradish peroxidas (HRP) conjugated $\mathrm{RBP}_{\lambda 03 \Delta 1-120}$ was added directly to inactivated blood (taken from the carcass' left nostril) (right reaction tube) as well as to inactivated sheep blood which served as a negative control (left reaction tube). After washing, chromogenic HRP substrate was added and color development photo-documented after $1 \mathrm{~min}$ B: Recombinant fusion protein mCherry-RBP $\mathrm{P}_{\lambda 03 \Delta 1-120} \mathrm{Was}$ added to $100 \mu \mathrm{l}$ of blood and directly subjected to fluorescence microscopy. Shown are merged 
images of transmission and fluorescent light (wavelengths: excitation $594 \mathrm{~nm}$, emission: $610 \mathrm{~nm}$ ). Scale bar: $5 \mu \mathrm{m}$. C: Dilutions of the inactivated cow blood (1-1:10, $2-1: 100,3-1: 1000)$ as well as dilutions of isolated DNA $(4-1: 10,5-1: 100,6-1: 1000)$ from the blood-samples were subjected to $16 S$ rRNA SNP-PCR. Shown are representative realtime PCR amplification-curves.

\section{B. anthracis strains BF-1 and BF-5 are clonal, very closely related outbreak strains}

Genomic DNA of $B$. anthracis strains BF- 5 was subjected to sequencing resulting in three contigs (chromosome, plasmid pXO1 and pXO2) (accession \# SRR16572036). Comparison of the genomes of B. anthracis strains BF-1 and BF-5 revealed that both strains were exceptionally similar (Table 1 ). The chromosome of BF-5 featured only three SNPs and two single nucleotide repeat (SNR) differences (both SNRs in non-coding regions with deletions of a single " $\mathrm{T}$ "). While plasmid pXO1 was identical, pXO2 harbored a single additional SNP- and SNR-insertion (" $\mathrm{T}$ ") in three identical repeat regions, respectively. This clonality of the two outbreak strains clearly supported the hypothesis that a hitherto non-localized source of unknown origin of contamination exists on-site. This source is very likely the cause of repeated infection of grassing cows on this pasture.

Table 1: DNA sequence differences between genomes of $B$. anthracis BF-1 and BF-5.

\begin{tabular}{|l|l|l|l|l|}
\hline Reference (BF-1) & Position & $\begin{array}{l}\text { BF-1 nucleotide } \\
\text { sequence }\end{array}$ & $\begin{array}{l}\text { BF-5 nucleotide } \\
\text { sequence }\end{array}$ & $\begin{array}{l}\text { Kind of } \\
\text { change }\end{array}$ \\
\hline $\begin{array}{l}\text { CP047131.1 } \\
\text { (chromosome) }\end{array}$ & 519877 & C & T & SNP (SNP1) \\
\hline CP047131.1 & 1434950 & $\begin{array}{l}\text { CTTTTTTTTTTTTTTGTAAA } \\
\text { TAA }\end{array}$ & $\begin{array}{l}\text { CTTTTTTTTTTTTTGTAAA } \\
\text { TAA }\end{array}$ & Deletion \\
\hline CP047131.1 & 1625072 & A & C & SNP (SNP2) \\
\hline CP047131.1 & 1878269 & $\begin{array}{l}\text { GTTTTTTTTTTTTTTGTAA } \\
\text { AATTAA }\end{array}$ & $\begin{array}{l}\text { GTTTTTTTTTTTTTTGTAA } \\
\text { AATTAA }\end{array}$ & Deletion \\
\hline CP047131.1 & 2472315 & T & C & SNP (SNP3) \\
\hline $\begin{array}{l}\text { CP047133.1 } \\
\text { (plasmid pX02) }\end{array}$ & $\begin{array}{l}29759 \\
31759\end{array}$ & CTTTTTTTAT & CTTTTTTTTAT & Insertion \\
\hline $\begin{array}{l}\text { CP047133.1 } \\
\text { (plasmid pX02) }\end{array}$ & 62640 & A & G & SNP (SNP4) \\
\hline
\end{tabular}

Phylogenetically strains B. anthracis BF-5 and -1 group with strains from the Austrian state of Tyrol

The canSNP-type of $B$. anthracis BF-5 was determined, assigning the strain to the B.Br.CNEVA clade [32]. Chromosomal sequence analysis inferred the phylogenetic placement of strain BF-5 to a cluster of central European B. anthracis strains within the B.Br.CNEVA clade. As expected from Table 1, the closest relative was strain BF-1 (Figure 3). Other close relatives were Tyrol 4675 and Tyrol 6282, from the Austrian state of Tyrol from 1988 and 1979, respectively. Strains from a large French B.Br.CNEVA cluster (only three representatives shown in Figure 3) as well as strains from Switzerland, Slovakia, Germany and Italy were more distantly related. Not shown are additional B.Br.CNEVA genomes phylogenetically more loosely related to the focus strain, BF-5. Notably, there is a polytomy at the base of the French cluster, the clade comprising strains A016/170D930 and Tyrol 3520 and the clade featuring BF-1, BF-5 and Tyrol 4674 and Tyrol 6282 (Figure 3). This clearly suggests a common ancestor of all the strains. 
A.

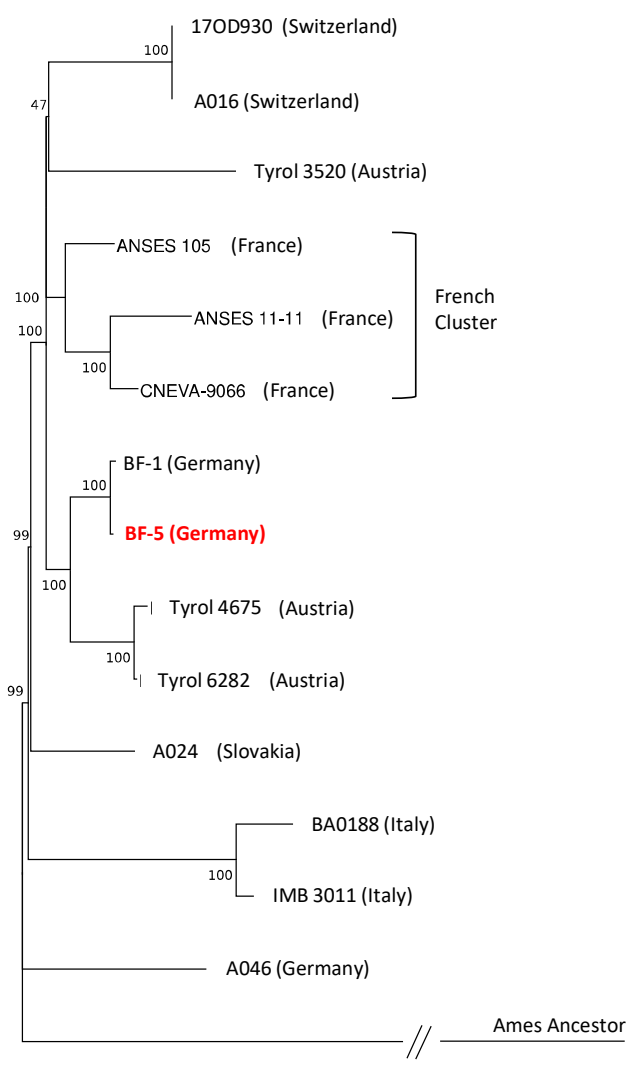

200

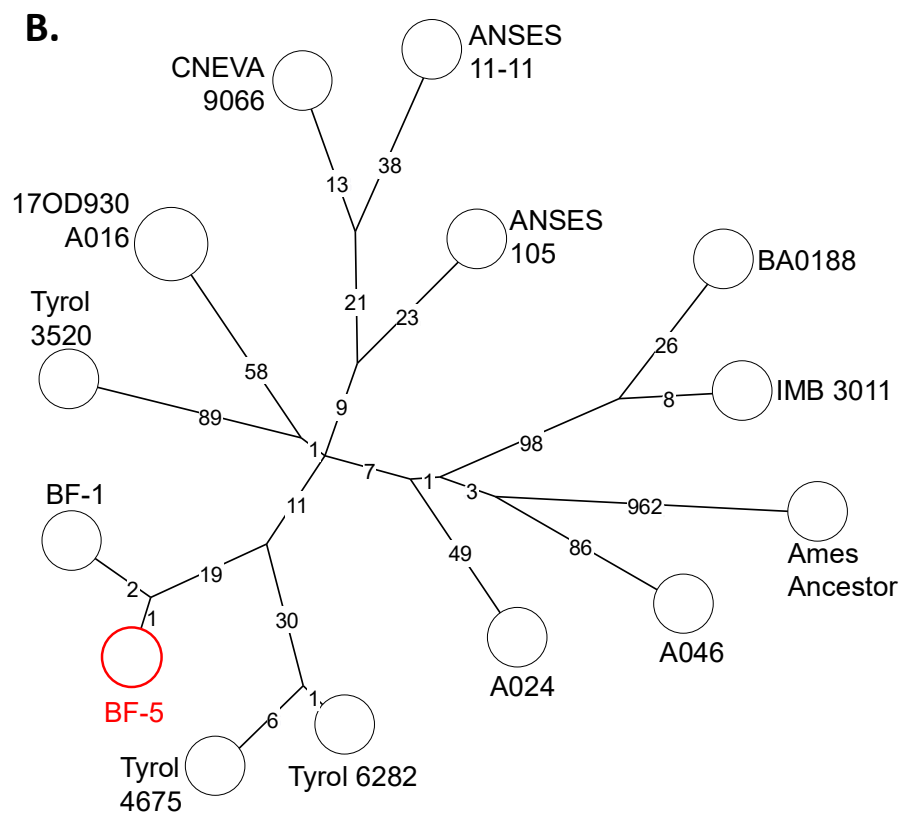


Figure 3: Phylogeny of new $B$. anthracis isolate BF-5 among its close relatives of the B.Br.CNEVA canonical SNP-clade. A rooted phylogenetic tree of representatives of the B.Br.CNEVA canonical single-nucleotide polymorphisms (canSNP) clade of $B$. anthracis is shown (A). The tree is based on 1558 chromosomal SNPs used to construct a Maximum Likelihood tree (bootstrap confidence from 500 permutations were generated and the tree with the highest likelihood is shown). Isolate names and countries of origin are indicated at branch termini (red: sequenced in this study; black:

sequences from public databases, Table S1). A Minimum-spanning tree of close relatives of strain BF5 within the B.Br.CNEVA canSNP-clade of B. anthracis derived from chromosomal SNPs is shown (B). Indicated are numerical SNP-differences (logarithmic scale) between chromosomes. Both trees are rooted to the reference chromosome, B. anthracis strain Ames 'Ancestor' that belongs to the A.Br.Ames canSNP-clade.

Both "classical", established methods and novel phage RBP reporter fusions enable direct detection and isolation of $B$. anthracis from soil-samples

Soil was retrieved from the site of the carcass as well as from surrounding areas up to $80 \mathrm{~m}$ away. (Figure 1A, B). The established analysis methods yielded positive PCR results after cultivation of original soil materials. Isolated colonies with typical morphology of $B$. anthracis were positive in PCR for pagA, capC and saspB (data not shown). The novel, phage protein-based magnetic enrichment approach fared equally well, yet, can be completed in much shorter time: To screen the possibly contaminated soil-samples for $B$. anthracis spores, $m$ Cherry-RBP ${ }_{\lambda 03 \Delta 1-120}$ was just added to soil supernatants pre-incubated with germination medium and the samples were subjected to fluorescence microscopy. With this method, cells of $B$. anthracis could be detected directly in soilsamples as cell chains emitted strong red fluorescence derived from the attached RBP reporter (Figure 4A). While presence of $B$. anthracis was indicated by fluorescence microscopy, isolation of $B$. anthracis from soil-samples was achieved using magnetic beads coupled with $\mathrm{RBP}_{\lambda 03 \Delta 1-120}$. After binding of the cells to the RBP-loaded magnetic beads, the buffer-washed cell-bead-complexes (Figure $4 \mathrm{~B}$, left panel) were agar-plated and cultured. A representative result is shown in Figure 4B (right panel). While occasionally hemolytic, non-B. anthracis colonies also grew on the plates, suspect B. anthracis colonies showing no hemolysis were chemically inactivated and confirmed by ELPRA (Figure 4C). Genomic DNA from six of these additional isolates was prepared for further analysis. 
Figure 4
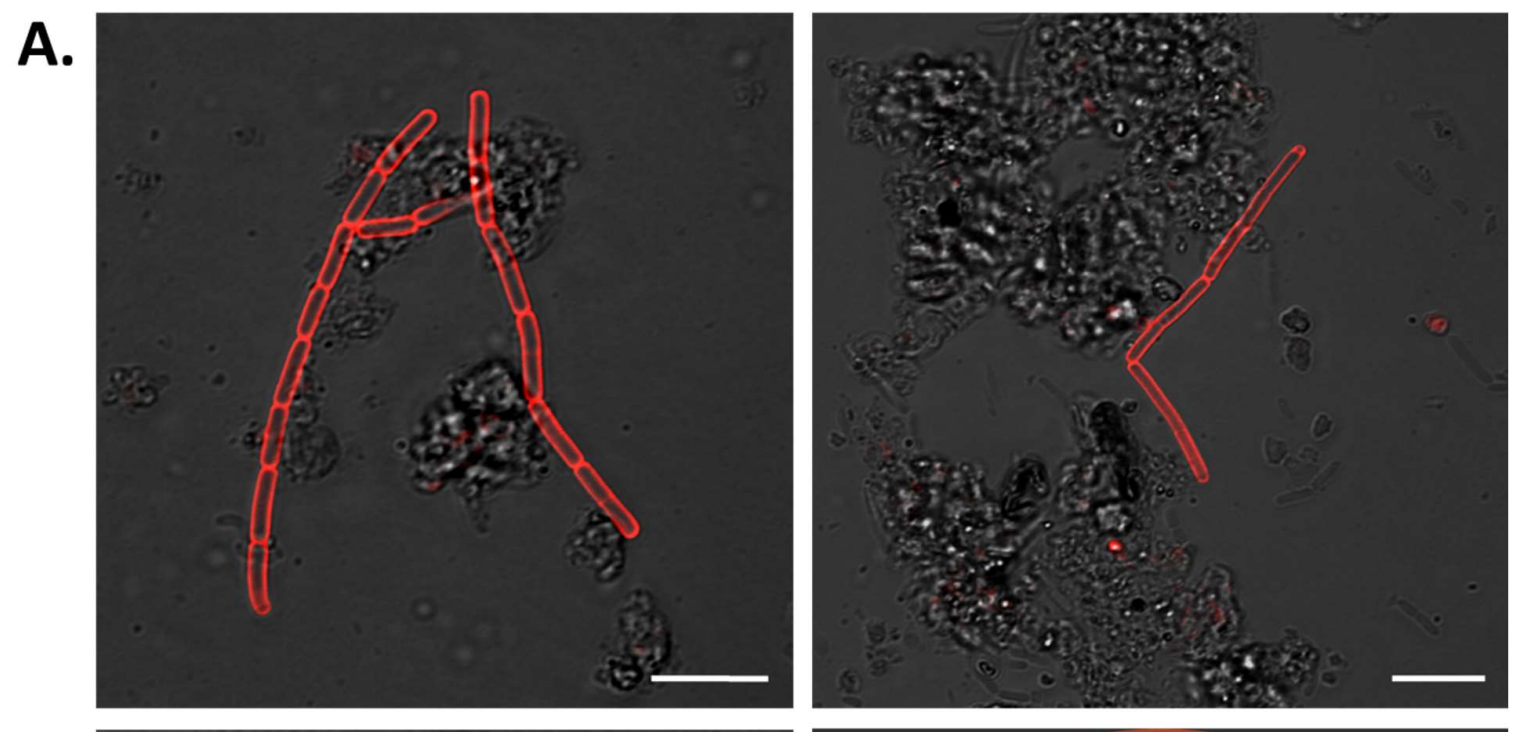

B.
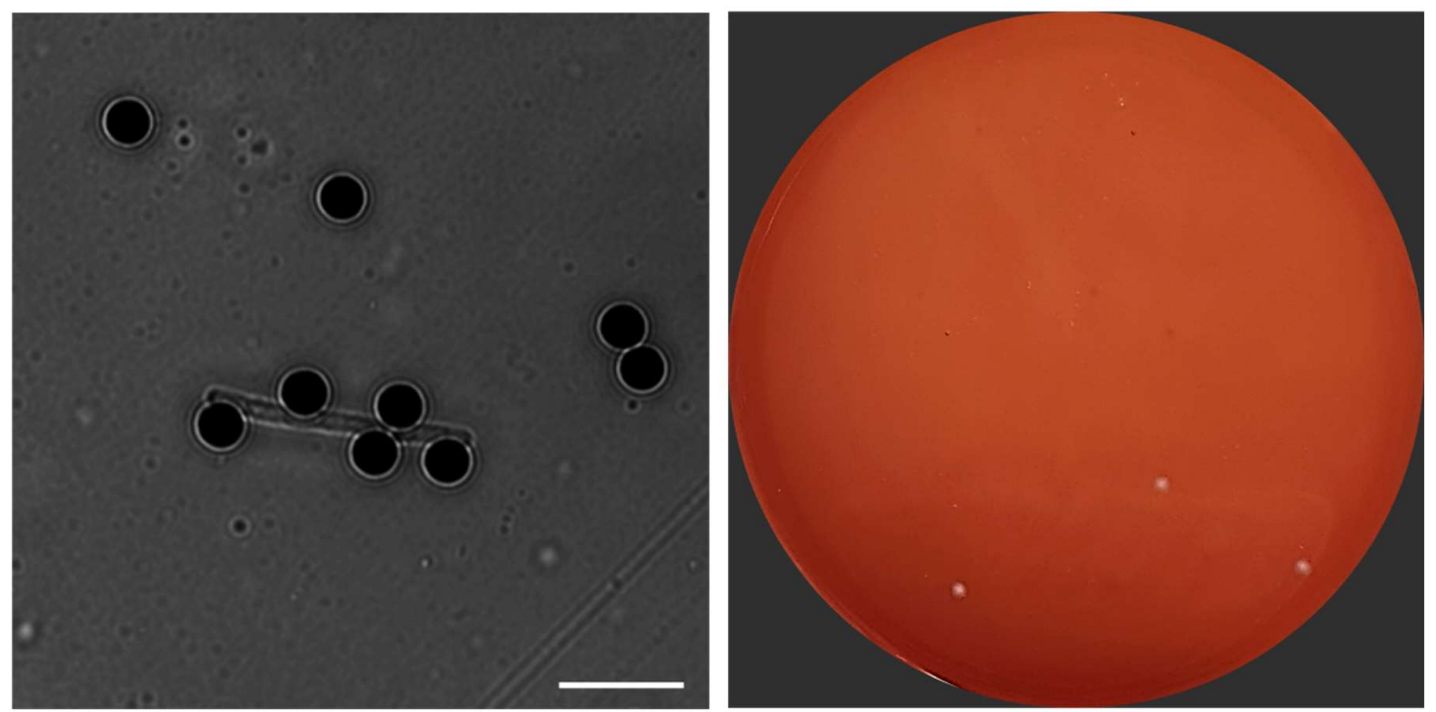

C.
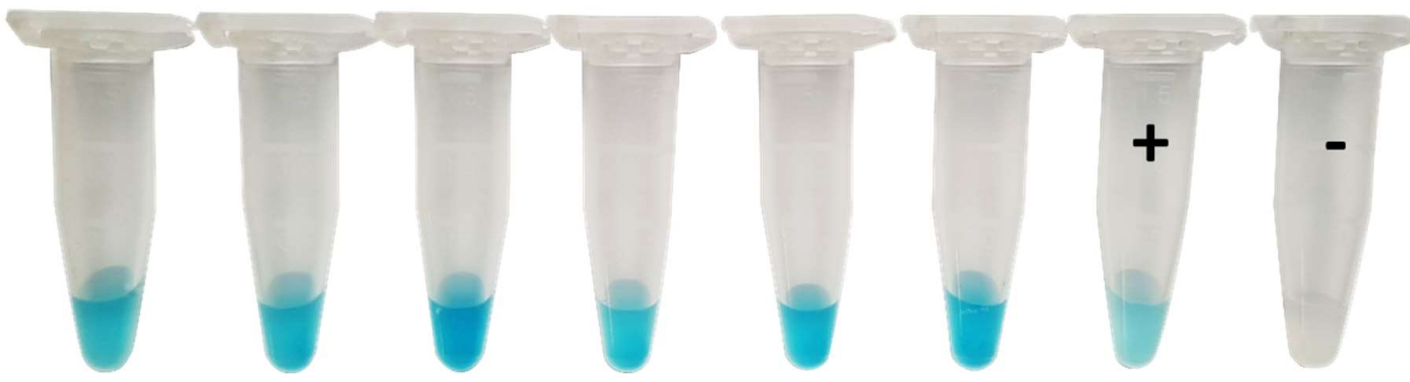

Figure 4: Direct detection and isolation of $B$. anthracis from contaminated soil-samples associated with a diseased cow. Soil-samples were shaken in PBST buffer to solubilize spores, centrifuged and the supernatant mixed with $\mathrm{BHI}$ broth containing fetal calf serum and incubated to allow spores to germinate. A: Recombinant fusion protein mCherry-RBP ${ }_{\lambda 03 \Delta 1-120}$ was added to pre-incubated soil supernatants and directly subjected to fluorescence microscopy. Shown are two merged images of transmission and fluorescent light (wavelengths: excitation $594 \mathrm{~nm}$, emission: $610 \mathrm{~nm}$ ). Scale bar: 5 $\mu \mathrm{m}$. B: Magnetic beads coupled with $\mathrm{RBP}_{\lambda 03 \Delta 1-120}$ were added to pre-incubated soil supernatants to 
capture B. anthracis cells. A sample was taken for brightfield microscopy (left panel, Scale bar: $5 \mu \mathrm{m}$ ) and the remainder of the bead suspension buffer-washed, plated on blood agar plates and incubated at $37^{\circ} \mathrm{C}$ overnight (right panel). C: Rapid RBP reporter-based assay on inactivated suspicious colony material from enrichment plates. Inactivated colony material was incubated with $\mathrm{RBP}_{\lambda 03 \Delta 1-120}$ covalently linked to horseradish peroxidase for colorimetric identification with chromogenic substrate. Positive control (+) was B. anthracis Sterne and negative control (-) B. cereus ATCC10987. Results were scored after about $1 \mathrm{~min}$ as positive (blue color development) or negative (no color development).

Four SNPs found between B. anthracis strains BF-1 and BF-5 were interrogated in additional isolates derived from contaminated soil

In order to determine the distribution and relative abundance of the four SNPs separating $B$. anthracis strains BF-1 and BF-5 (Table1; Table S2), PCRs of the identified four SNP-regions were conducted on DNA from six soil isolates and the PCR amplicons Sanger-sequenced. We did not identify any SNP-differences in these six soil isolates relative to BF-5 (data not shown). Thus, these results indicate that the BF-5 genotype is the prevalent genotype at the affected pasture in 2021.

\section{Discussion}

Regarding risk-assessment, re-occurrence of an anthrax-outbreak after 12 years [9] at the same pasture diminished the suspicion of intentional release of the pathogen as underlying cause. Conversely, the outbreak strongly indicated that an old anthrax focus was still active. This is reminiscent to similar situations in other regions of Europe. For instance, in Sweden an outbreak in cattle occurred in a nature-reserve in 2011. Notably, records positioned an old anthrax burial-site (mid-1940s) in that area [2,33]. Remarkably, only two years later, an additional cow deceased closely to this area that had been seen cattle-vaccination after the 2011 outbreak [34]. Similar to the case at hand, genome sequencing of the two Swedish outbreak isolates from 2011/2013 indicated these were clonal [2]. The authors offered as plausible explanation for this genomic identity among spatially and temporally separated outbreaks the spreading of spores by birds or wildlife. Though these Swedish outbreaks have caused public alarm for the risk of environmental contamination [2], no more cases were reported in that region since (as of November 2021). More active is the reemerging situation in Italy where anthrax resurfaces repeatedly in the southern region of Basilicata $[35,36]$ and soils at outbreak -sites remained contaminated with viable spores for many years $[4,5]$.

The genomes of strains BF-1 and BF-5 differ by only three chromosomal SNPs (Table 1). A recent genomic study on an anthrax-outbreak in Italy found strains differing by up to five SNPs [37]. Genome analysis for epidemiologic investigation of strains associated with injectional anthrax have led the authors to the conclusion that genetic variation is possibly generated as a result of infection of a single host but some phylogenetic patterns might be best explained by diversity introduced through several infection-cycles of B. anthracis in several hosts [8]. The 2021 outbreak in Bavaria seems to follows this pattern with only very few SNP-differences between strains from the same outbreak-site separated by 12 years. Notably, all six isolates retrieved from soil surrounding the carcass-site and from $80 \mathrm{~m}$ away at a ditch featured the same unique SNP-positions as isolate BF-5 directly grown from the dead cow's blood. In contrast, it is very unlikely that isolate BF-1 is a direct ancestor of BF-5. Chromosomal SNP 1 differs from the ancestor-state only in BF-5 but not in BF-1. Vice versa, however, chromosomal SNP 2 and SNP 3 showed an evolved state in BF-1, while ancestral in BF-5 (Table 1). 
In order to acutely diminish the local risk of surface-near spore contamination on-site, the affected pasture-site where the animal fell (Figure $1 \mathrm{~A}$ and $\mathrm{B}$ ), was disinfected with $10 \mathrm{l} / \mathrm{m}^{2} 10 \%(\mathrm{v} / \mathrm{v})$ formaldehyde as similarly advised by [1]. Obviously, this measure will neither be able disinfect deeper soil horizons nor eliminate the unidentified original contamination-site presumably located somewhere on the premises. Longer term monitoring of surface-near soil on-site may be able to alert authorities in case B. anthracis spores can again be detected after favorable weather conditions, e.g., heavy rains followed by mild temperatures [38]. Further developments related to sensitive detection of $B$. anthracis in soil could facilitate the identification and elimination of the original source of spore contamination at the affected premises.

In any case, this rare outbreak provided an ideal opportunity for real-life testing of assays developed beforehand for detection and identification of $B$. anthracis. Direct microscopy of $B$. anthracisinfected blood (Figure 2A) or germinated cells in $B$. anthracis spore-contaminated soil (Figure 4A) and rapid testing of inactivated blood (Figure 4B) or suspect colonies [23] yielded similar results with this authentic materials to previously tested spiked-in materials (unpublished).

\section{Acknowledgements}

The authors thank Malena Bestehorn-Willmann for help with genome sequencing, Rahime Terzioglu, Gabriele Echle, Linda Dobrzykowski, and Laura Madeddu for technical assistance.

\section{Funding}

This research work was funded by the Medical Biological Defense Research Program of the Bundeswehr Joint Medical Service (to GG and MA) and was partially funded through Bundeswehr Medical Service [SoFo 56Z1-S-43 1922] to MA and the German Federal Ministry of Education and Research (BMBF) [ZooSeq FKZ 01KI1905A] to MA.

\section{Conflicts of Interest}

The authors declare no conflicts of interest. Opinions, interpretations, conclusions, and recommendations are those of the authors and are not necessarily endorsed by any governmental agency, department, or other institutions. The funders had no role in the design of the study; in the collection, analyses, or interpretation of data; in the writing of the manuscript, or in the decision to publish the results.

\section{Data Availability Statement}

All data produced in the present study are available upon reasonable request to the authors. 


\section{References}

1. Turnbull PC. World Health Organization. Anthrax in humans and animals. Geneva (CH): WHO Press, 2008.

2. Ågren J, Finn M, Bengtsson B, Segerman B. Microevolution during an anthrax outbreak leading to clonal heterogeneity and penicillin resistance. PLoS One 2014; 9:e89112.

3. Revich BA, Podolnaya MA. Thawing of permafrost may disturb historic cattle burial grounds in East Siberia. Global Health Action 2011; 4.

4. Fasanella A, Di Taranto P, Battisti A, et al. Old animal anthrax outbreaks discovered through the analysis of soil. Giornale Italiano die Medicina Tropicale 2011; 16:1-4.

5. Braun P, Grass G, Aceti A, et al. Microevolution of anthrax from a young ancestor (M.A.Y.A.) suggests a soil-borne life cycle of Bacillus anthracis. PLoS ONE 2015; 10:e0135346.

6. Hanczaruk M, Reischl U, Holzmann T, et al. Injectional anthrax in heroin users, Europe, 20002012. Emerg Infect Dis 2014; 20:322-3.

7. Ringertz $\mathrm{SH}$, Hoiby $\mathrm{EA}$, Jensenius $\mathrm{M}$, et al. Injectional anthrax in a heroin skin-popper. Lancet 2000; 356:1574-5.

8. Keim $P$, Grunow R, Vipond R, et al. Whole genome analysis of injectional anthrax identifies two disease clusters spanning more than 13 years. EBioMedicine 2015; 2:1613-1618.

9. Antwerpen M, Proenca DN, Ruckert C, et al. Draft genome sequence of Bacillus anthracis BF-1, isolated from Bavarian cattle. J Bacteriol 2012; 194:6360-1.

10. Antwerpen M, Elschner M, Gaede W, Schliephake A, Grass G, Tomaso H. Genome sequence of Bacillus anthracis strain Stendal, isolated from an anthrax outbreak in cattle in Germany. Genome Announc 2016; 4:e00219-16.

11. Elschner MC, Busch A, Schliephake A, Gaede W, Zuchantke E, Tomaso H. High-quality genome sequence of Bacillus anthracis strain 14RA5914 isolated during an outbreak in Germany in 2014. Genome Announc 2017; 5.

12. Price EP, Seymour ML, Sarovich DS, et al. Molecular epidemiologic investigation of an anthrax outbreak among heroin users, Europe. Emerging Infectious Diseases 2012; 18:1307-1313.

13. BAYERISCHE, LANDESTIERÄRZTEKAMMER. Aktueller Fall von Milzbrand bei Weiderindern in Bayern. BLTK Newsletter 2009; 3:1.

14. Braun $P$, Knupfer $M$, Antwerpen $M$, Triebel $D$, Grass $G$. A rare glimpse into the past of the anthrax pathogen Bacillus anthracis. Microorganisms 2020; 8.

15. Derzelle S, Aguilar-Bultet L, Frey J. Whole genome SNP analysis of bovine B. anthracis strains from Switzerland reflects strict regional separation of Simmental and Swiss Brown breeds in the past. Vet Microbiol 2016; 196:1-8.

16. Sterne M. Anthrax. In: Stableforth AW, Galloway IA, eds. Infectious Diseases of Animals, Disease due to Bacteria. London: Butterworth, 1959: 16-52. 
17. Fasanella A, Di Taranto P, Garofolo G, et al. Ground Anthrax Bacillus Refined Isolation (GABRI) method for analyzing environmental samples with low levels of Bacillus anthracis contamination. BMC Microbiol 2013; 13:167.

18. Braun $P$, Wolfschläger I, Reetz $L$, et al. Rapid microscopic detection of Bacillus anthracis by fluorescent receptor binding proteins of bacteriophages. Microorganisms 2020; 8:934.

19. Braun P, Nguyen MD-T, Walter MC, Grass G. Ultrasensitive detection of Bacillus anthracis by real time PCR targeting a polymorphism in multi-copy $16 \mathrm{~S}$ rRNA genes and their transcripts. medRxiv 2021; [preprint], 2021.09.20 [cited 2021 Oct 26], 2021.09.20.21263746.

20. Beyer W, Bellan S, Eberle G, et al. Distribution and molecular evolution of Bacillus anthracis genotypes in Namibia. PLoS Negl Trop Dis 2012; 6:e1534.

21. Turnbull, Peter C. Anthrax in Humans and Animals. 4th ed. Geneva: World Health Organization, 2008. Available at: http://www.ncbi.nlm.nih.gov/books/NBK310486/. Accessed 5 October 2021.

22. Rohde A, Papp S, Feige P, Grunow R, Kaspari O. Development of a novel selective agar for the isolation and detection of Bacillus anthracis. J Appl Microbiol 2020;

23. Braun P, Rupprich N, Neif D, Grass G. Enzyme-Linked Phage Receptor Binding Protein Assays (ELPRA) Enable Identification of Bacillus anthracis Colonies. Viruses 2021; 13:1462.

24. Knüpfer M, Braun $\mathrm{P}$, Baumann K, et al. Evaluation of a highly efficient DNA extraction method for Bacillus anthracis endospores. Microorganisms 2020; 8.

25. Antwerpen MH, Zimmermann P, Bewley K, Frangoulidis D, Meyer H. Real-time PCR system targeting a chromosomal marker specific for Bacillus anthracis. Mol Cell Probes 2008; 22:313-5.

26. Kolmogorov M, Yuan J, Lin Y, Pevzner PA. Assembly of long, error-prone reads using repeat graphs. Nat Biotechnol 2019; 37:540-546.

27. Angiuoli SV, Gussman A, Klimke W, et al. Toward an online repository of Standard Operating Procedures (SOPs) for (meta)genomic annotation. OMICS 2008; 12:137-141.

28. Lärkeryd A, Myrtennäs K, Karlsson E, et al. CanSNPer: a hierarchical genotype classifier of clonal pathogens. Bioinformatics 2014; 30:1762-4.

29. Treangen TJ, Ondov BD, Koren S, Phillippy AM. The Harvest suite for rapid core-genome alignment and visualization of thousands of intraspecific microbial genomes. Genome Biol 2014; 15:524.

30. Tamura K, Nei M. Estimation of the number of nucleotide substitutions in the control region of mitochondrial DNA in humans and chimpanzees. Mol Biol Evol 1993; 10:512-26.

31. Kumar S, Stecher G, Li M, Knyaz C, Tamura K. MEGA X: Molecular evolutionary genetics analysis across computing platforms. Mol Biol Evol 2018; 35:1547-1549.

32. Van Ert MN, Easterday WR, Huynh LY, et al. Global genetic population structure of Bacillus anthracis. PLoS One 2007; 2:e461.

33. Lewerin SS, Elvander M, Westermark T, et al. Anthrax outbreak in a Swedish beef cattle herd-1st case in 27 years: Case report. Acta Vet Scand 2010; 52:7. 
34. Elvander M, Persson B, Sternberg Lewerin S. Historical cases of anthrax in Sweden 1916-1961. Transbound Emerg Dis 2017; 64:892-898.

35. Fasanella A. Bacillus anthracis, virulence factors, $\mathrm{PCR}$, and interpretation of results. Virulence 2013; 4.

36. Fasanella A, Garofolo G, Galante D, et al. Severe anthrax outbreaks in Italy in 2004: considerations on factors involved in the spread of infection. New Microbiol 2010; 33:83-6.

37. Abdel-Glil MY, Chiaverini A, Garofolo G, et al. A whole-genome-based gene-by-gene typing system for standardized high-resolution strain typing of Bacillus anthracis. J Clin Microbiol 2021; 59:e0288920.

38. Van Ness GB. Ecology of anthrax. Science 1971; 172:1303-7. 